\title{
Analysis and Performance of Lumped-Element Kinetic Inductance Detectors for W-band
}

\author{
Beatriz Aja, Marina C. de Ory, Luisa de la Fuente, Member IEEE, Eduardo Artal, Life Member IEEE, \\ Juan Pablo Pascual, Senior Member IEEE, María Teresa Magaz, Daniel Granados, Alicia Gomez
}

\begin{abstract}
Lumped-Element Superconducting resonators are a promising technology for its use in millimeter-wave observations and quantum computing applications that require large arrays of extremely sensitive detectors. Among them, Lumped-Element Kinetic Inductor Detectors (LEKIDs) have shown good performance in the submillimeter band in several Earth-based telescopes. In this work, LEKIDs for their use as millimeter-wave receivers of astronomical applications are presented. LEKIDs arrays using a thin bilayer of superconducting titanium/aluminum (Ti/Al), deposited on silicon substrate, have been designed and fabricated. The design of a dual-polarization LEKID with the goal of detection at $\mathrm{W}$-Band for two orthogonal polarizations is described and a fabricated array has demonstrated absorption at ambient temperature. Also, an approximate design methodology of the coupling parameter for LEKIDs readout, essential for dynamic range optimization of the detector under millimeter wave radiation, is proposed. In addition, resonance characteristics and coupling factor of the fabricated superconducting resonators using high-quality internal factor $Q_{i}$ under cryogenic temperatures have been analyzed. The design guidelines in this work are applicable to other LEKIDs arrays, and the presented superconducting Ti/Al thin film LEKIDs can be used in future receiver arrays in millimeter bands.
\end{abstract}

Index Terms - Kinetic Inductance Detector, superconducting microwave devices, lumped-element resonator, cryogenics, millimeter wave astronomy, polarimetry.

\section{INTRODUCTION}

$\mathrm{K}$ INETIC Inductance Detectors (KIDs) are used in high sensitivity radio astronomy receivers. These detectors operate under the property of inductance change of a superconducting strip, when it is cooled down well below its superconducting critical temperature, usually near absolute zero, when they absorb a microwave signal taken by a telescope receiver system. KIDs based receivers are built containing thousands of individual detectors, or instrument pixels, as their intrinsic multiplexing is a relatively simple way of receiver integration. Frequency domain multiplex operation consists of

Manuscript received July 22, 2020. This work was supported by Ministry of Science, Innovation and Universities under Grants ESP2017-83921-C2-2-R, ESP2017-86582-C4-1-R, ESP2017-86582-C4-3-R, ESP2017-92706-EXP, AY A2017-92153-EXP, "Severo Ochoa” Programme for Centres of Excellence in R\&D (MINECO, Grant SEV-2016-0686). By Comunidad de Madrid under Grant P2018/NMT-4291. D.G. and A.G also acknowledge Grant DEFROST N62909-19-1-2053 from ONRGlobal. A.G. acknowledges IJCI-2017-33991. This paper is an expanded version from the IEEE MTT-S International Microwave Symposium (IMS 2020), Virtual Conf., August 4-6, 2020 (Corresponding authors: Beatriz Aja and Alicia Gomez). a high amount of resonant circuits coupled to a single readout transmission line, making feasible such a high number of instrument pixels relatively easy [1]. Each individual KID is embedded in a resonant circuit having a resonant frequency different from the remainder KIDs. In this way, the readout system is in fact a frequency division multiplex communication system [2]. Former KID receivers operated in submillimeter bands, but, nowadays, the use of new superconducting materials, allows detection in the optical-near IR band [3] or even in the millimeter band [4],[5] showing good performance in all cases.

KIDs are based on superconducting microresonators with a characteristic resonant frequency and quality factor. The absorption of an incoming radiation, with energy higher than the superconducting gap, changes the kinetic inductance $\left(\mathrm{L}_{\mathrm{k}}\right)$, leading to a change of the resonant frequency. Moreover, this absorption also produces dissipative losses in the resonator, decreasing the quality factor in comparison with the darkness condition. The reduction of resonant frequency and quality factor, given proper calibration, enables the detection of radio signals from astronomical sources, employing complex processing algorithms.

Several KID radio astronomy instruments for millimeter and submillimeter wave observations are in operation, achieving even thousands of multiplexed detectors [6]-[10]. There are two main types of detectors: Antenna coupled- Microwave Kinetic Inductance Detectors (MKIDs) [2] and Lumped Element Kinetic Inductance Detectors (LEKIDs) [11]. The former are based on distributed resonators with coplanar-waveguide (CPW) geometries coupled to an antenna that acts as an active receiver. The later, LEKIDs, which are the focus of our study, are based on lumped inductor-capacitor resonators, where the inductor acts directly as the effective optical absorber, and its design is shown in Fig. 1.

B. Aja, L. de la Fuente, E. Artal and J.P. Pascual are with Dpt. Ing. Comunicaciones, University of Cantabria, 39005 Santander, Spain (e-mail: ajab@unican.es).

M.C. de Ory is with Centro de Astrobiología (CSIC-INTA), Torrejón de Ardoz, E-28850 Madrid, Spain and with IMDEA-Nanociencia, Cantoblanco, E-28049 Madrid, Spain.

M.T. Magaz, A. Gomez are with Centro de Astrobiología (CSIC-INTA), Torrejón de Ardoz, E-28850 Madrid, Spain (email: a.gomez@cab.inta-csic.es).

D. Granados is with IMDEA-Nanociencia, Cantoblanco, E-28049 Madrid, Spain (e-mail: daniel.granados@imdea.org). 


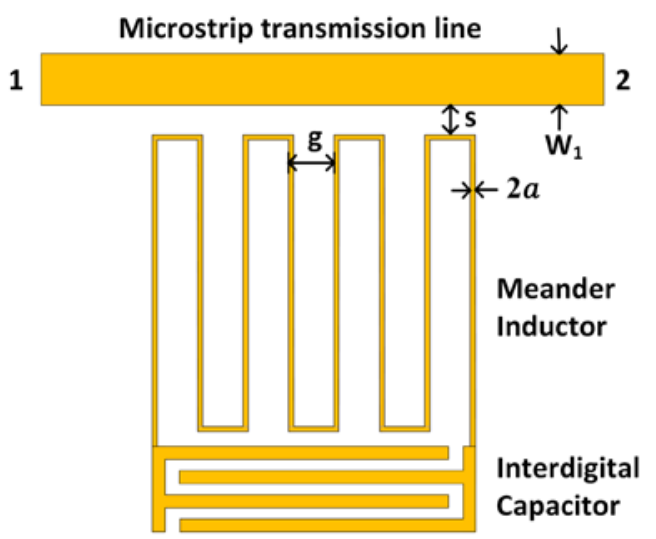

Fig. 1. Structure of a LEKID (Lumped Kinetic Inductance Detector): Meander Inductor with strips of width $2 a$ and spacing $g$. Coupling separation $s$ between the resonator and the readout $50 \mathrm{Ohm}$ microstrip feedline of width $W_{1}$.

Great efforts have been made to develop dual-polarization LEKIDs and to improve the sensitivity at W-band for future Cosmic Microwave Background observations. For instance, A. Catalano et al. [5], [12], [13], showed that the superconducting proximity effect in a titanium/aluminum (Ti/Al) bilayer causes a decrease in the superconducting critical temperature that pushes the LEKID detection to the W-band. Regarding polarization sensitivity, earth-based instruments such as NIKA2 [9] use an external polarizer that separates the two linear polarizations into two independent arrays based on a Hilbert fractal structure. On the other hand, some additional works have focused on the development of dual-polarization LEKIDs demonstrating simultaneous orthogonal polarization sensitivity in the millimeter and submillimeter wave ranges [8], [14]. However, very little has been done in developing dualpolarization LEKIDs in the W-band. Therefore, in this work, we focus on both requirements and present an optimized version of the BiKID approach [15] for the W-band, based on an optimized optical coupling design.

The response of LEKIDs is maximized when critical coupling is achieved, i.e. the external quality factor $\left(Q_{c}\right)$ equals the internal quality factor $\left(Q_{i}\right)$ [16]. Whereas $\mathrm{Q}_{\mathrm{i}}$ is set by fixed parameters such as the optical background or operating base temperature, $Q_{c}$ can be modified by tuning geometrical parameters as separation $s$. Therefore, a design methodology for the microwave coupling to the LEKID has been developed and applied to a new prototype, with several external factors $Q_{c}$.

This paper presents the analysis, design, fabrication and experimental tests of bilayer LEKID prototypes working in Wband ( 75 to $110 \mathrm{GHz}$ ). The remainder of this article is organized as follows. Section II deals with the KID design, concerning millimeter wave signals to be detected, and low frequency resonators design, with special focus on external coupling. Section III describes fabrication and prototypes assembly. Section IV presents experimental systems to perform ambient temperature and cryogenic tests. Tests results are detailed in Section V. Finally, Section VI presents conclusions summarizing this paper.

\section{Lumped-ElEMENT Kinetic Inductance Detector DESIGN}

The presented LEKIDs are based on series superconducting capacitor-inductor resonators coupled to a single transmission line, as it was first proposed in [11]. The inductor, which acts as the effective absorber, is designed to absorb the incident radiation by matching optically to free space. In this case, the strip grating, deposited on top of a high resistive silicon substrate, was designed to be matched at $\mathrm{W}$ band to the freespace impedance, when a backshort is placed at the rear part of the silicon wafer. The linearly polarized wave absorption of this design is characterized elsewhere and peaks around $78 \mathrm{GHz}$ [17].

A new dual-polarization LEKID is designed to absorb and distinguish millimeter-wave radiation of two orthogonal linearly polarized waves simultaneously. For this purpose, two LEKIDs, based on the design explained before, can be stacked one on top of the other with perpendicular orientation as shown in Fig. 2. In this case, each of them will be dedicated to the detection of one of the two perpendicular polarizations of the incident waves.

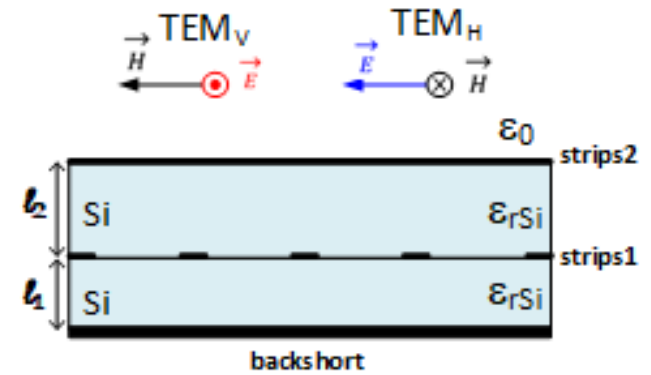

Fig. 2. Cross section of a dual-polarization LEKID on silicon substrate.

\section{A. Mm-wave Coupling Design}

An equivalent circuit model of the dual-polarization LEKID is shown in Fig. 3.

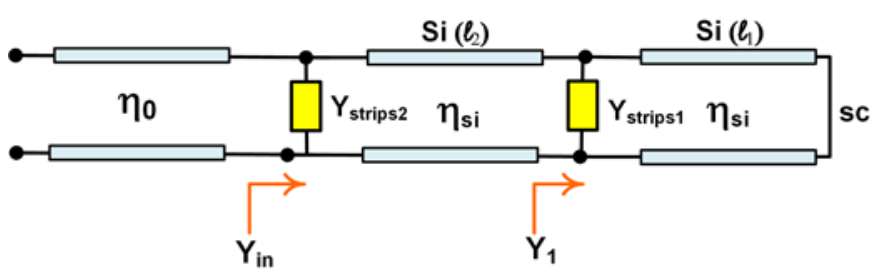

Fig. 3. Equivalent circuit to model a dual-polarization LEKID. Short-circuit (SC) termination accounts for the presence of a backshort. The shunt admittances $Y_{\text {strips1 }}$ and $Y_{\text {strips2 }}$ are the equivalent circuits of the strips.

The input admittance of the dual-polarization LEKID is

$$
Y_{\text {in }}=Y_{\text {strips } 2}+\frac{1+\tanh \left(\gamma \ell_{2}\right) / \eta_{s i} Y_{1}}{\eta_{s i} \tanh \left(\gamma \ell_{2}\right)+1 / Y_{1}}
$$

where the admittance $Y_{1}$ is given by

$$
Y_{1}=Y_{\text {strips } 1}+\frac{1}{\eta_{s i} \tanh \left(\gamma \ell_{1}\right)}
$$


and $\gamma=\alpha_{S i}+j \beta_{S i}$ is the complex propagation constant, where the real part is the attenuation constant $\alpha_{S i}$, due to dielectric losses, and the imaginary part $\beta_{S i}$ is the phase constant in silicon. The shunt admittances $Y_{\text {strips } 1}$ and $Y_{\text {strips2 } 2}$ are the equivalent circuits of the strips that compose the inductors of the LEKIDs. Silicon substrates thicknesses are $\ell_{1}$ and $\ell_{2}$. Silicon wave impedance is $\eta_{S i}=\eta_{0} / \sqrt{\varepsilon_{r S i}}$ with $\varepsilon_{r S i}=11.9$ and $\eta_{0} \approx 120 \pi$ Ohm.

The strips that compose the LEKID inductors have width $2 a$, distance between strips $g$ and a sheet resistance $R_{s}(\mathrm{Ohm} / \mathrm{sq})$. It is worth to note that even though the detector is operating at temperatures well below its critical temperature $(T c)$, an incident radiation with frequency $(v)$ that provides energy higher than the superconducting gap ( $\Delta$ ), i.e. $h v>2 \Delta$, where $h$ is the Planck constant, leads to a sheet resistance comparable to that in normal conducting state just above the superconducting critical temperature [18].

Metallic strips present a resistive and inductive impedance for an incident electric field parallel to them [18]-[20], which admittance is given by

$$
Y_{\text {StripsL }}=1 /\left(R_{s} \frac{g}{2 a}+j X_{L}\right)
$$

where the reactance $X_{L}$ is

$$
X_{L}=\eta_{0} \frac{g}{\lambda_{0}} \ln \left(\csc \left(\pi \frac{a}{g}\right)\right)
$$

If the incident electric field is orthogonal to the strips, the equivalent circuit is a capacitive admittance, according to [21, Sec. 5.18, eq.(1a)].

A dual-polarization LEKID with two stacked and orthogonal strips is shown in Fig. 4. The admittance $Y_{\text {in }}$ in (1) depends on incident wave polarization shown in Fig. 2. The admittance $Y_{\text {strips } 1}$ is inductive as (3) and $Y_{\text {strips2 } 2}$ is capacitive in case of a wave TEM $\mathrm{V}_{\mathrm{V}}$ On the other hand, admittances $Y_{\text {strips1 }}$ and $Y_{\text {strips2 }}$ are capacitive and inductive as (3) respectively for $\mathrm{TEM}_{\mathrm{H}}$.

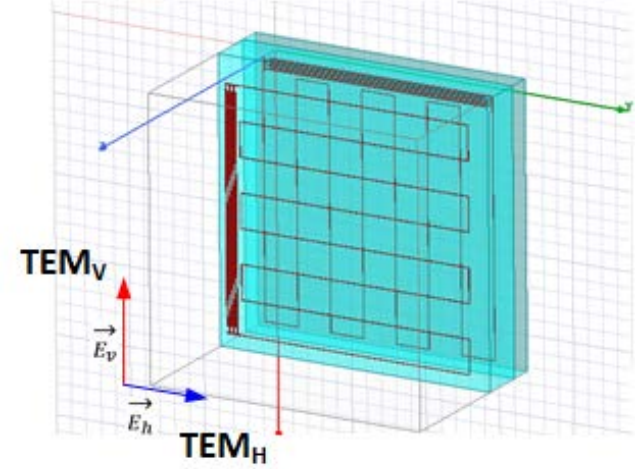

Fig. 4. Dual-polarization LEKID and two incident TEM waves with electric fields orthogonal between them. Each LEKID is composed of a meander line inductor and an interdigital capacitor.

The strip is designed to achieve efficient absorption, therefore, for an inductive strip admittance in (3), the geometry $(a, g)$ is calculated to fulfill $\operatorname{real}\left(Y_{\text {stripsL }}\right)=1 / \eta_{0}$ with metal resistance $R_{s}\left(\frac{O h m}{s q}\right)$ at cryogenic temperature. Then the grounded silicon substrate presents the imaginary admittance required to match the imaginary part of the admittance in (3) at the center frequency for a thickness $\ell_{1}$ given by

$$
\ell_{1}=\frac{1}{\beta_{S i}}\left(\pi-\cot ^{-1}\left(\frac{\eta_{S i} X_{L}}{\left(R_{s} \frac{g}{2 a}\right)^{2}+X_{L}^{2}}\right)\right)
$$

The second LEKID formed by rotating the same strips geometry $90^{\circ}$ with respect to the previous one on silicon substrate, with thickness a half wavelength at the center frequency, which allows matching simultaneously in both strips. This type of structure has narrow bandwidth behavior, since impedance matching is achieved with one single silicon dielectric layer $\ell_{1}$.

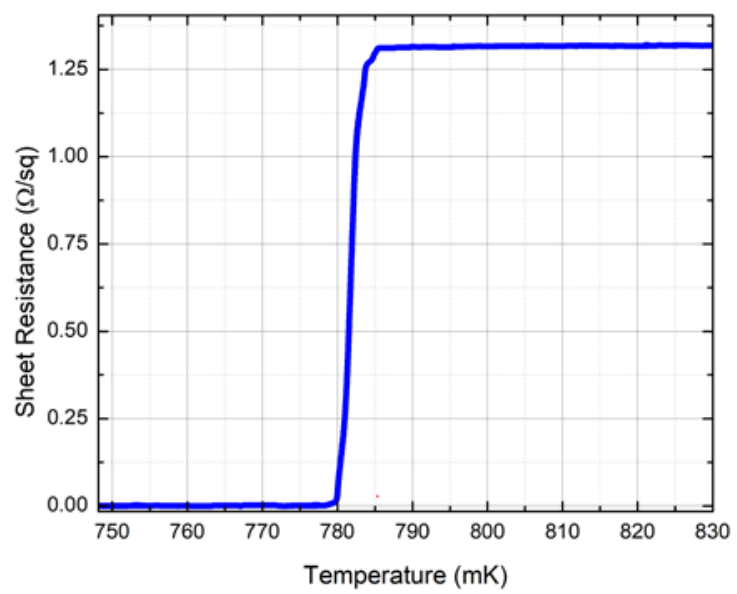

Fig. 5. Sheet resistance versus temperature of a thin titanium/aluminum (Ti/Al) bilayer sample.

The initial geometry of the strips for the LEKIDs design has been obtained for a $35 \mathrm{~nm}$ thick Ti/Al film with $R_{s}=1.27$ Ohm/sq measured just above Tc (see Fig. 5). A strip width $2 a$ $=3 \mu \mathrm{m}$ has been chosen to achieve the broadest bandwidth around $90 \mathrm{GHz}$, which together with a spacing $g=375 \mu \mathrm{m}$ provides $\operatorname{real}\left(Y_{\text {StripsL }}\right)=1 / \eta_{0}$ in (3). Using those values, the inductive and capacitive admittances are calculated. Their reflection coefficients on air, from 65 to $110 \mathrm{GHz}$, are depicted in Fig. 6 (a).

The thickness $\ell_{1} \approx 292 \mu \mathrm{m}$ of the grounded silicon substrate is calculated using (5), and the silicon substrate thickness between LEKIDs is $\ell_{2}=\lambda_{\mathrm{Si}} / 2 \approx 483 \mu \mathrm{m}$ with $\lambda_{S i}=\lambda_{0} / \sqrt{\varepsilon_{r s i}}$, both calculated at $90 \mathrm{GHz}$. The obtained reflection coefficients, of the dual-polarization LEKID model in Fig. 2, for the two linear polarized incident waves $\left(\mathrm{TEM}_{\mathrm{V}}, \mathrm{TEM}_{\mathrm{H}}\right)$ and lossless silicon substrate, are plotted in Fig. 6(b) from 65 to $110 \mathrm{GHz}$, showing a perfect matching and maximum absorption at 90 $\mathrm{GHz}$. The loss tangent of high resistive silicon in millimeter bands at $4.2 \mathrm{~K}$ is around $1.12 \cdot 10^{-6}$ [22], therefore it has been considered negligible for the design. Moreover, the obtained 
impedance matching, for both incident waves, is not affected by the strip with capacitive performance.

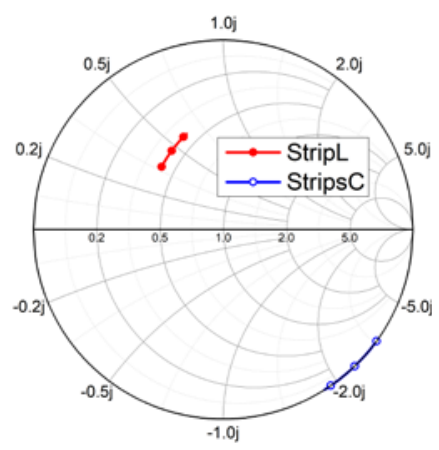

(a)

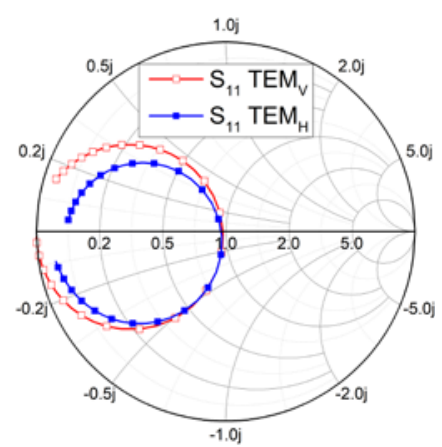

(b)
Fig. 6. Ti/Al film $35 \mathrm{~nm}$ thick strips with $R s=1.27 \mathrm{Ohm} / \mathrm{sq}, 2 a=3 \mu \mathrm{m}$ and $g=375 \mu \mathrm{m}$ in the frequency band $65-110 \mathrm{GHz}$ : (a) Reflection coefficient of the strips on air, for incident linearly polarized waves parallel and orthogonal to the strips (b) Input reflection coefficient of the dual-polarization LEKID for both incident waves TEM.

With this initial design, the next step is a 3D electromagnetic simulation at W-band of the dual-polarized LEKID using the HFSS 3D electromagnetic simulator from ANSYS, as a single unit cell with Floquet ports with master and slave boundaries, to simulate an array as a planar-periodic structure. The final millimeter wave absorption, of the dual-polarization LEKID, for the two linearly polarized incident waves, is also simulated with the complete structure composed of the meandered strips and interdigital capacitors (see Fig. 4), and it is shown in [17]. The absorption efficiency in each component, using the Field Calculator available in the HFSS 3D electromagnetic simulator, is shown in Fig. 7 for both polarizations. This tool makes use of the Surface Loss function to estimate the energy absorbed in both inductors and capacitors in the LEKID [23]. The simulation results show a total absorption efficiency of $98 \%$ for each mode, showing the inductors the highest absorption efficiency with $\sim 90 \%$.

\section{B. Low frequency Resonator Design}

A typical LEKID superconducting resonator is coupled to the main microstrip readout line as it is shown in Fig. 1. The interdigital capacitor fixes its resonant frequency and allows frequency multiplexing. For readout, the resonator is coupled to a transmission line, selecting the coupling coefficient with a separation $s$ (see Fig. 1). Absorption of photons at millimeter wave results in a change of resonator frequency and quality factor, therefore an accurate design of the coupling is crucial for dynamic range optimization of the detector under millimeter wave radiation.

The connection of inductive and capacitive parts forms a series RLC resonant circuit. The resistive part of this equivalent circuit comes from conductor losses of both inductive and capacitive parts. As we are dealing with superconducting resonators, this resistive part can be extracted from the twofluid model and tends to zero when the operating temperature is much lower than its critical temperature [11].

LEKID resonators are absorption-mode coupled [24], and the effect of an individual resonator on readout line transmission is only present in a narrow band around its resonant frequency. Fig. 8 shows an equivalent circuit with two coupled resonators in a transmission test schematic [25].

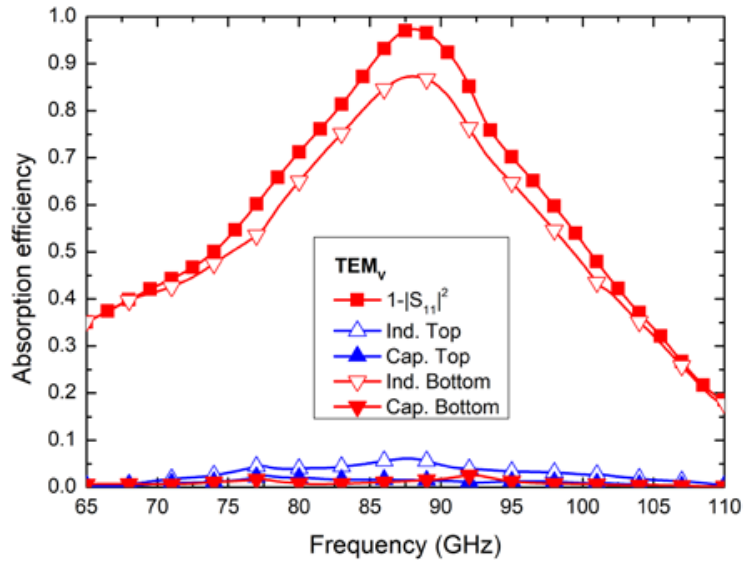

(a)

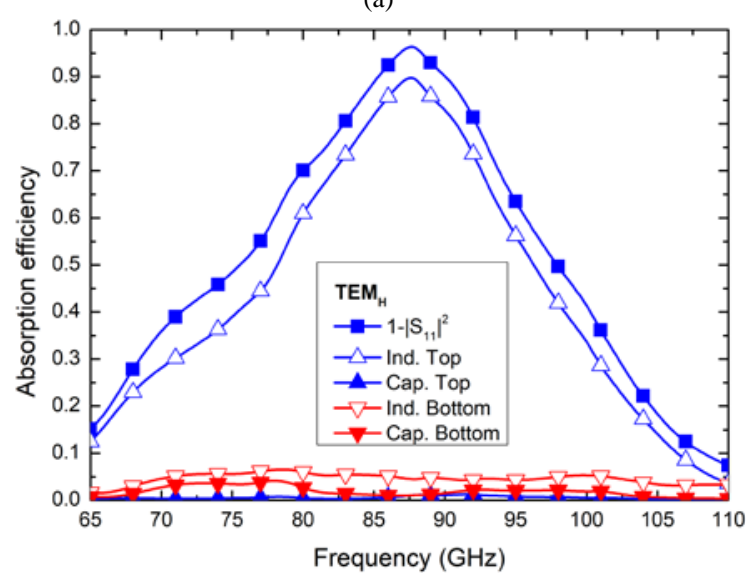

(b)

Fig. 7. Simulated absorption efficiency of each component, inductors (Ind.) and capacitors (Cap.), and total absorption $\left(1-\left|\mathrm{S}_{11}\right|^{2}\right)$ for the dual-polarization LEKID; (a) $\mathrm{TEM}_{\mathrm{V}}$ wave, with maximum absorption in the LEKID at the bottom. (b) $\mathrm{TEM}_{\mathrm{H}}$ wave with maximum absorption in the LEKID at the top.

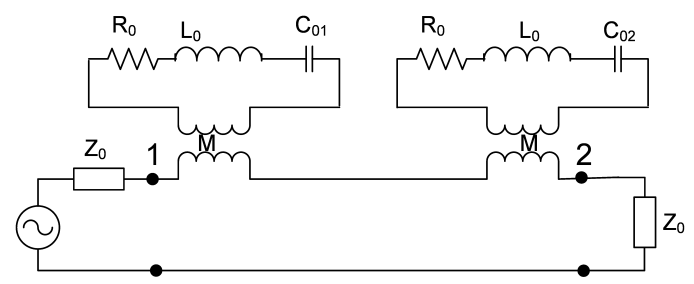

Fig. 8. Equivalent circuit of two coupled resonators, in absorption mode, to the readout line. $\mathrm{M}$ is the mutual inductance. $\mathrm{R}_{0}, \mathrm{~L}_{0}, \mathrm{C}_{0 \mathrm{i}}$ represent the equivalent series resonant circuit of a LEKID.

Mutual inductance $M$ is the main coupling effect, which transforms each series-resonant circuit to a shunt-resonant circuit. In a narrow frequency band, taking into account only one resonator and considering other resonances well detuned, the equivalent circuit shown in Fig. 9 is useful to analyze LEKID resonator quality factors and coupling factor, and to obtain their relation to the $S_{21}$ scattering parameter. This last transmission parameter is in fact the tested one in a LEKIDs instrument. 


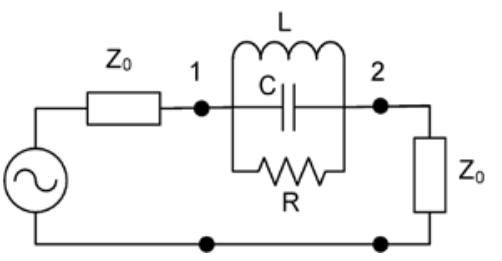

Fig. 9. Equivalent circuit of a LEKID resonator coupled to the readout line. R, L, C are transformed shunt resonant circuit elements.

Coupling factor $k$ is the relation between delivered power to external loads $P_{E}$ and internally delivered power $P_{0}$ inside the resonator, at resonant frequency, given by

$$
k=\frac{P_{E}}{P_{0}}=\frac{R}{2 Z_{0}}=\frac{Q_{i}}{Q_{c}}
$$

where $Q_{i}$ is the internal quality factor and $Q_{c}$ the external quality factors [26]. For critical coupling, $k$ is 1 and the power delivered to the resonator is maximum.

LEKID coupling is analyzed as a pair of asymmetric coupled microstrip lines, having a total coupling length $\ell$ equal to the contribution of meander line sections close to the readout line: $\ell=4 \cdot(g+2 a)$, see Fig. 1 . In a typical LEKID this coupling length is very short in terms of wavelength at the resonant frequency $\left(f_{0}\right)$, and the equivalent circuit can be analyzed as a differential length $(d z)$ of a coupled lines pair, as it is shown in Fig. 10(a). All elements $\left(C_{1}, C_{2}, C_{M}, L_{1}, L_{2}\right.$ and $\left.M\right)$ have dimensions per unit of length. Readout microstrip line width $\left(W_{1}\right)$ is typically chosen to have $50 \mathrm{Ohm}$ characteristic impedance, whereas inductor strip width $W_{2}=2 a$ is narrow in order to optimize the LEKID millimeter wave absorption, as well as to achieve an inductive value adequate for the resonant frequency, inside the selected radiofrequency band for the readout.

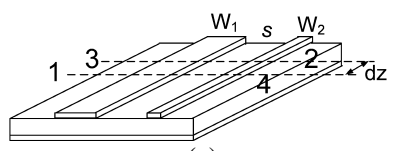

(a)

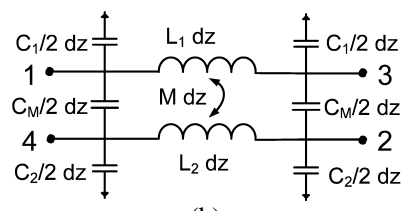

(b)
Fig. 10. (a) Asymmetric coupled microstrip lines. (b) Equivalent circuit of a differential section $(d z)$.

Performing a circuit analysis of the schematic shown in Fig. 10(b), using typical values for the microstrip structure, it is straightforward to realize that the most relevant element in the coupling is mutual inductance $M d z$. The equivalent circuit can be greatly simplified for design purposes, since all capacitive elements have a negligible effect. Using the equivalent circuits shown in Fig. 8 and Fig. 9, the mutual inductance required to obtain a specific coupling for a LEKID resonator is:

$$
M_{\ell}=M \cdot \ell=\frac{1}{\omega_{0}} \sqrt{R R_{0}}
$$

where $\omega_{0}$ is the resonant angular frequency $2 \pi f_{0}$.

Coupling design can be simplified using symmetry properties of a two-port network [27]. Symmetry axis of a
LEKID coupled resonator is depicted in Fig. 11. Applying odd mode condition, a short circuit in all points of the symmetry axis, the two-port network is converted to a one-port network, which is applied to the coupled lines network. Moreover, due to symmetry properties, the LEKID impedance $Z_{K I D}$, the inductance elements in mutual inductance equivalent network and the coupling length are halved. The resultant equivalent networks are shown in Fig. 12(a) and Fig. 12(b). These two networks have identical $Y$ parameters, being their calculation the first step for the proposed design process. By analysis of mutual inductance circuit in Fig. 12(b) its $Y_{21}$ parameter is

$$
Y_{21}=-j \frac{\omega_{0} \frac{M_{\ell}}{2}}{\omega_{0}^{2}\left[\left(\frac{M_{\ell}}{2}\right)^{2}-\frac{L_{1 \ell}}{2} \frac{L_{2 \ell}}{2}\right]}
$$

For a specific LEKID coupling design, the input data are: dielectric substrate parameters, the width of coupled lines, $W_{1}$ and $W_{2}=2 a$ in Fig. 10(a), the coupling length $\ell$ and the resonant frequency $\mathrm{f}_{0}$. Given the input data, and after selecting the desired coupling level: overcoupling, undercoupling or critical coupling, the mutual inductance $\mathrm{M}_{\ell}$ is calculated according to (7). Inductances $L_{1 \ell}$ and $L_{2 \ell}$ in (8) are calculated from inductances per unit length $L_{1}$ and $L_{2}$ of isolated microstrip lines, for widths $W_{1}$ and $W_{2}$. The assumption of isolation of microstrip lines, for $L_{1}$ and $L_{2}$ calculation purposes, is a good approach, because coupling of LEKID resonators is very weak in general, and both lines are separated by a sufficiently large distance $s$.

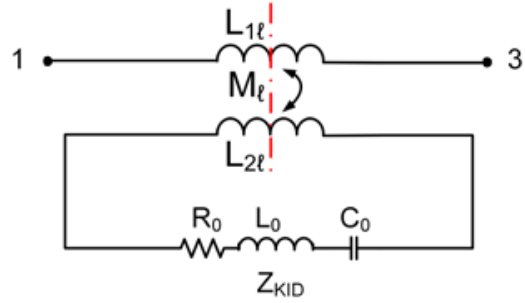

Fig. 11. Symmetry axis in a LEKID coupled network.

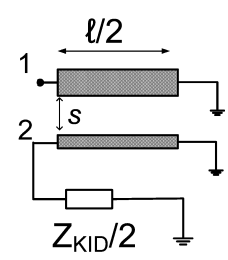

(a)

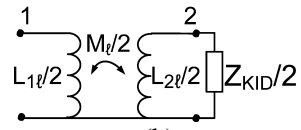

(b)

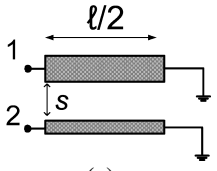

(c)
Fig. 12. Equivalent networks obtained by odd mode symmetry condition applied to the LEKID coupled resonator: (a) Coupled lines network, (b) Mutual inductance equivalent network. Port 2 is terminated with a half of LEKID resonator impedance, (c) Asymmetric coupled microstrip lines for electrical circuit simulation of odd mode condition.

The next step is to obtain the right separation $s$ between lines using an asymmetrical coupled microstrip lines electrical model [28]. This model is available in microwave circuit simulators, and through an optimization routine, or by manual tuning, the separation $s$ value must be varied to achieve the desired $Y_{21}$ parameter, of the two-port network in Fig. 12(c), with the value 
calculated in (8) at the resonant frequency $\omega_{0}$. For an improved accuracy of the separation $s$, a 2D electromagnetic simulator is used, taking as an initial value the obtained s value by electrical model simulation, to avoid the uncertainties of coupled lines electrical models for very weak couplings and short coupling lengths.

For the readout design of a LEKID, applying the present design method, Table I shows the obtained separation s for three different coupling factors regarding a LEKID with an expected internal quality factor $Q_{i}=200000$ and resonant frequency $f_{0}=$ $500 \mathrm{MHz}$. It has been considered a high resistive silicon substrate with thickness $285 \mu \mathrm{m}$, coupling length $\ell=4 \cdot(g+2 a)$ $=3 \mathrm{~mm}$, and line widths $W_{1}=220 \mu \mathrm{m}$ for $50 \mathrm{Ohm}$ readout line, $W_{2}=2 a=3 \mu \mathrm{m}$ for inductor strip. The $\mathrm{S}_{21}$ responses of a LEKID for the three coupling factors $(k)$ with the parameters obtained in Table I are simulated and the results are shown in Fig. 13.

TABLE I

CALCULATED SEPARATION S AND LOADED QUALITY FACTOR $Q$, FOR THREE COUPLING FACTORS $(k)$. LEKID WITH $Q_{I}=200000 \mathrm{AND} f_{0}=500 \mathrm{MHz}$.

\begin{tabular}{lcccc}
\hline Coupling & $\boldsymbol{k}$ & $\left|\mathbf{S}_{\mathbf{2 1}}\right|^{2} \min (\mathbf{d B})$ & $\boldsymbol{s}(\boldsymbol{\mu m})$ & $\boldsymbol{Q}$ \\
\hline \hline over & 3 & -12.0 & 323 & 50000 \\
critical & 1 & -6.0 & 505 & 100000 \\
under & 0.25 & -1.94 & 787 & 160000 \\
\hline
\end{tabular}

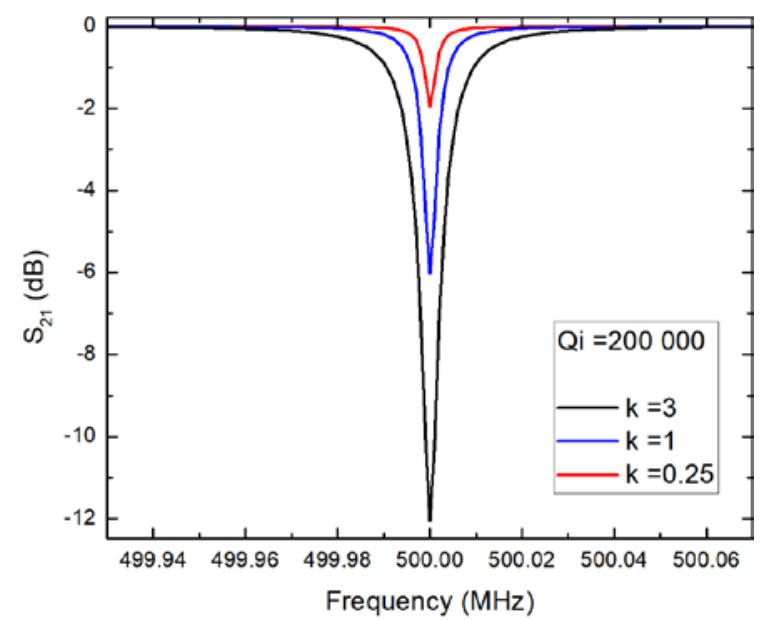

Fig. 13. Simulated $\mathrm{S}_{21}$ response of a LEKID for three coupling factors (k) with the parameters shown in Table 1.

\section{KIDs FABRICATION AND ASSEMBLY}

The devices were fabricated following the technological process detailed in [17]. In summary, by means of confocal sputtering, a Ti/Al bilayer was deposited on a high resistive silicon wafer $275 \mu \mathrm{m}$ thick, with a $200 \mathrm{~nm}$ thick Al layer on the rear part of the wafer, to be used as ground plane and optical backshort. The physical dimensions for the inductor were calculated considering an initial approach of $R_{s} \frac{g}{2 a} \cong 377 \mathrm{Ohm}$ in (3). The LEKIDs were designed for single polarization, and two prototypes were fabricated and measured at cryogenic and ambient temperatures with $2 a=3 \mu \mathrm{m}$ and $g=440 \mu \mathrm{m}$ for optical coupling. The bilayer was characterized at cryogenic temperatures, obtaining $R_{s}=1.27 \mathrm{Ohm} / \mathrm{sq}, T_{c}=782 \mathrm{mK}$ and an estimation of the kinetic inductance $L_{k}=2.24 \mathrm{pH} / \mathrm{sq}$. Preliminary results for low frequency cryogenic characterization, and optical absorption for single polarization, were presented in [17].

In order to verify the performance of a dual-polarization prototype, several stacked wafers were measured at ambient temperature as a proof of concept. An array of 11 x 11 singlepolarization LEKIDs was fabricated on a $275 \mu \mathrm{m}$ thick silicon wafer, while an identical second one was fabricated without a ground plane. Both wafers were stacked orthogonally with a $275 \mu \mathrm{m}$ thick silicon wafer in between, to distinguish the polarization between two linear polarized incident waves. Fig. 14(a) shows a photograph of the stacked wafers placed in a test fixture, to characterize them at ambient temperature, whereas a photograph of the LEKID inductor is depicted in Fig. 14(b).

On the other hand, a new prototype with seven pixels was designed and fabricated modifying the coupling factor $Q_{c}$ between pixels. Separation $s$ and orientation of each LEKID, with respect to the single $50 \mathrm{Ohm}$ microstrip transmission line, were modified from pixel to pixel in order to tune external coupling $Q_{c}$. This device has been designed following the methodology explained in Section II.B, which is crucial for future designs, enabling the optimized critical coupling, i.e., $Q_{i}$ to be close to $Q_{c}$ under the desired optical load [16]. Fig. 15(a) and Fig. 15(b) show the design of two pixels, where their coupling has been modified by rotating $90^{\circ}$ the original pixel orientation, increasing the total coupling length $\ell$. In the remaining pixels, coupling has been modified by tuning the separation s between lines. This prototype was mounted inside a light-tight package made from bulk aluminum, where aluminum wirebonds were used to connect microstrip lines to the readout chain as shown in Fig. 15(c).

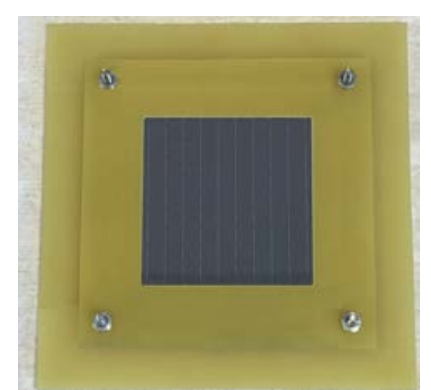

(a)

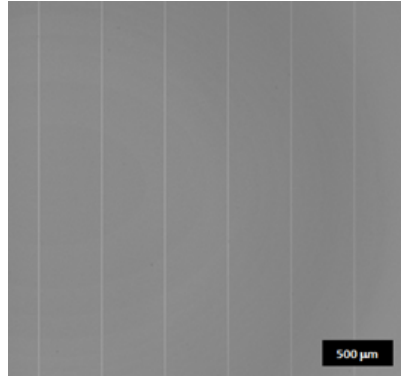

(b)
Fig. 14. Photograph of the dual-polarization prototype; (a) $11 \times 11$ LEKIDs for test at ambient temperature. Size of the sample $40 \mathrm{~mm}$ x $40 \mathrm{~mm}$; (b) Detail of a LEKID inductor. 

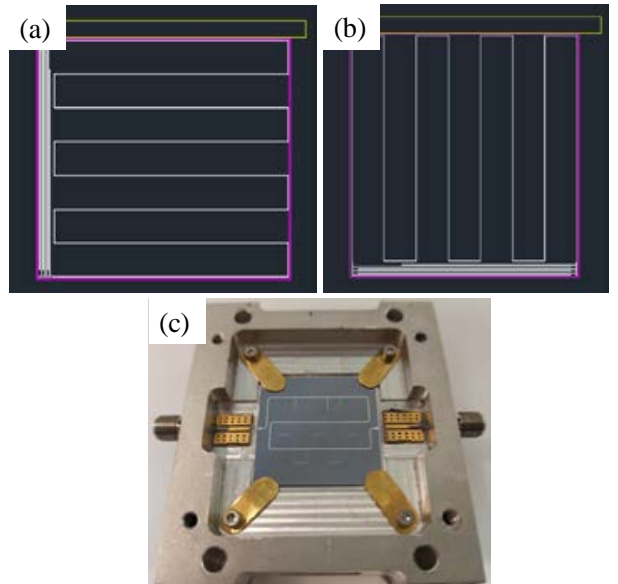

Fig. 15. (a) and (b) show schemes of two of the pixels designed for coupling tuning. (c) Fabricated LEKIDs array mounted for cryogenic testing.

\section{EXPERIMENTAL SYSTEMS}

This section describes two experimental test set-ups used for KIDs characterization: A W-band ambient temperature quasioptical system used for millimeter wave absorption test and a cryogenic test system to measure LEKIDs resonant frequencies and quality factors.

\section{A. Ambient Temperature Test System}

The ambient temperature test system operates at W-band. It has been set up in order to characterize the LEKIDs absorption through a free-space measurement. The system consists of two horn antennas, two dielectric lenses that collimate the beam at the measurement plane, and a vector network analyzer [29], [30], [17]. The quasioptical system has a $4 f$ topology, with $f$ the focal length $75 \mathrm{~mm}$ of PTFE plano-convex lenses LAT075 (Thorlabs), shown in Fig. 16. The horn antennas (QSH-SL-75110-F-20) provide around $20 \mathrm{~dB}$ gain. They are rectangular horns with dimensions $a \times b=14.8 \mathrm{~mm} \times 11 \mathrm{~mm}$ at the aperture, where the beam radii that maximize the coupling to the fundamental Gaussian mode are $\omega x=0.35 a, \omega y=0.5 b$ [30]. The beam-waist radius at $z=0$ is similar for both coordinates $b \omega r_{0} \approx 4.72 \mathrm{~mm}$. The lenses diameter is $D=50 \mathrm{~mm}$ and the beam radius at the antenna aperture is $\omega \approx 5.37 \mathrm{~mm}$, since $D>$ $4 \omega$, the fractional power lost is lower than $3.10^{-4}$ for the fundamental Gaussian mode [30]. The calculated output beam waist radius, at the middle of the system, is $b \omega r_{0, \text { out }}=16.87 \mathrm{~mm}$, which defines the size of the beam spot for the absorption measurement of LEKID arrays at ambient temperature.

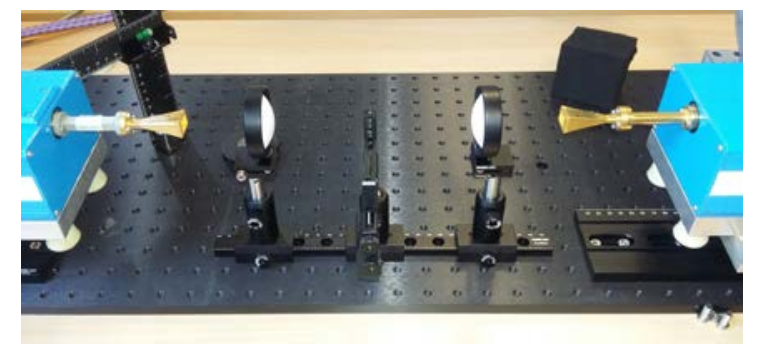

Fig. 16. W-Band quasioptical system with $4 f$ topology ( $f=75 \mathrm{~mm})$. Mm-wave Extension modules V10VNA2 WR-10 (OML), horn antennas and PTFE lenses.

\section{B. Cryogenic Test System}

The experimental cryogenic system is the BlueFors Dilution Refrigerator LD-250 shown Fig. 17. This cryostat consists of a dilution refrigerator (DR) backed by a two-stage pulse tube cooler that provides $60 \mathrm{~K}$ and $4 \mathrm{~K}$ temperature stages, while the DR provides a $0.7 \mathrm{~K}$ stage and a variable $12 \mathrm{mK}-1 \mathrm{~K}$ stage, where the detectors are mounted, Fig. 17(a). Electrical cryogenic characterization has been performed using the following read-out system, which connects the external warm electronics with the LEKID array at cryogenic temperatures.
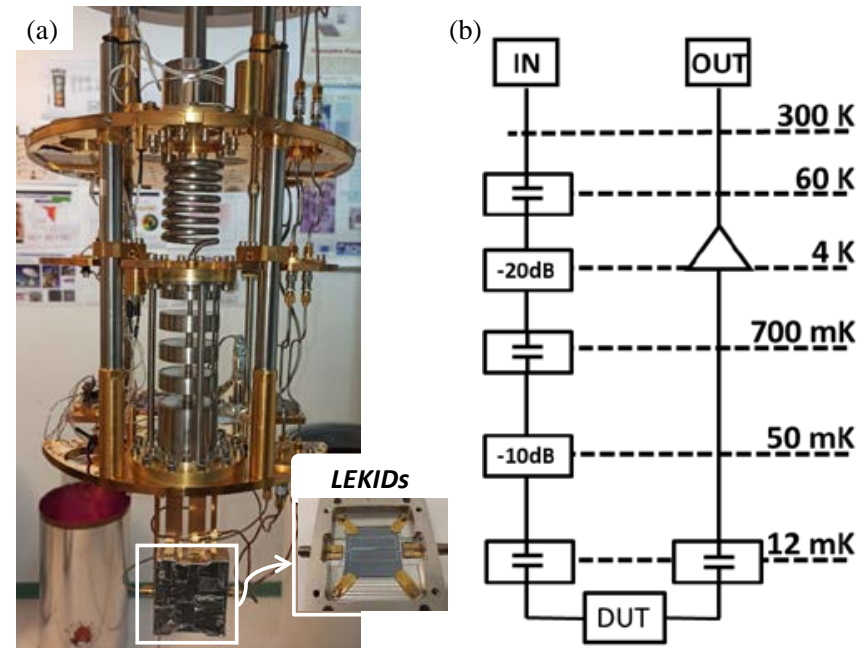

Fig. 17. (a) Dilution Refrigerator (DR) with the array mounted in a light-tight holder. Inset shows the LEKIDs device. (b) Schematic of the cabling configuration within the DR where the device under test (DUT) corresponds to the LEKIDs array.

\section{1) Cryogenic Harness}

The cryogenic harness scheme is shown in Fig. 17, which has been carefully chosen in order to minimize thermal loading between stages. Stainless steel inner and outer conductor coaxial cable is used from room temperature to the $4 \mathrm{~K}$ stage, being thermalized in the $60 \mathrm{~K}$ stage by a DC block. A $20 \mathrm{~dB}$ attenuator reduces the $300 \mathrm{~K}$ radiation and dissipates the power in the $4 \mathrm{~K}$ stage. Cupronickel (CuNi) coaxial is used from the 4 $\mathrm{K}$ stage to the $12 \mathrm{mK}$ stage. Again, two DC Blocks and an extra $10 \mathrm{~dB}$ attenuator reduces the noise contribution from the warmer stages. Finally, a semirigid copper coaxial cable connects the last DC-Block with the LEKID package. Aluminum wirebonds are used to connect a microstrip board to the LEKID chip. On the return path, a copper coaxial cable connects the package with a DC Block at the $12 \mathrm{mK}$ stage. Then, a superconducting NbTi coaxial cable carries the signal from this stage up to the $4 \mathrm{~K}$ stage where a SiGe Low Noise Amplifier (Caltech-CITLF3) amplifies the signal (15 dB gain). Finally, CuNi coaxial cable carries the signal to the output-port of the cryostat.

\section{2) Readout system}

In order to measure the transmission characteristic of the LEKID array, a readout system has been assembled in a chassis. It is composed of several coaxial modules such as SPDT (Single Pole Double Thru), LNA (Low Noise Amplifier), Attenuator, Power Splitter and Quadrature Modulator. Four SPDT RF 
Switches enable switching between Vector Network Analyzer (VNA) connection and the I/Q demodulator, through a Measurement Mode Option and it also switches between two different DUTs (Device Under Test) through a Channel Selection Option. The VNA measurements work from $40 \mathrm{MHz}$ to $2.6 \mathrm{GHz}$, and $\mathrm{I} / \mathrm{Q}$ demodulation measurements from 500 $\mathrm{MHz}$ to $2.6 \mathrm{GHz}$.

For I/Q measurements, the LO signal is generated by a commercial PSG Analog Signal Generator, and is split using power splitter. A step attenuator is used as a variable attenuator to tune the readout signal power level to the required level for the LEKIDs. The signal coming from the LEKID cryostat output is amplified with a gain block consisting of three amplifiers ( $\sim 43 \mathrm{~dB}$ gain), to reach the RF power required by an $\mathrm{I} / \mathrm{Q}$ demodulator. The block diagram of the readout is in Fig. 18. The "IN" and OUT" ports are connected to the cryogenic set-up shown in Fig. 17.

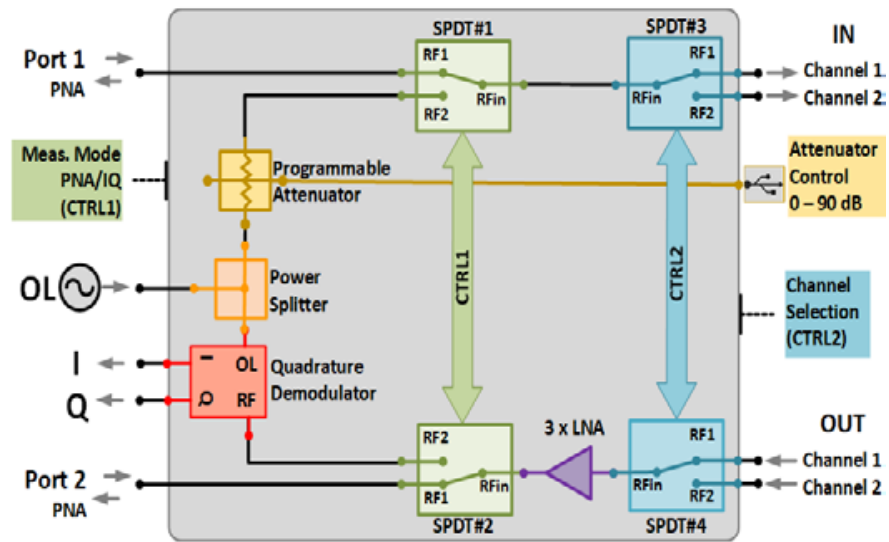

Fig. 18. Readout block diagram for measuring the resonances of a LEKID array.

\section{RESULtS}

The measurements described in the following section refer to the fabricated prototypes that have been detailed in Section IV. Measurements at ambient and cryogenic temperatures have been carried out, and their results have been compared with the simulations.

\section{A. Measurements at Ambient Temperature}

The dual-polarization prototype, made up of $11 \times 11$ LEKIDs, was characterized at ambient temperature. This prototype was measured for two orthogonal and linearly polarized waves in the 65 to $110 \mathrm{GHz}$ frequency band. Results are depicted in Fig. 19. These measurements were made using the quasioptical test-bench described in Section IV. A photograph of the sample under test is shown in Fig. 20. Simulations using the obtained parameters for the Ti/Al bilayers at ambient temperature $\left(R_{S}=4 \mathrm{Ohm} / \mathrm{sq}\right)$ are also included. The actual tested silicon substrate height $(h=295 \mu \mathrm{m})$ confirms a good fitting between simulated and experimental results for both polarizations, showing a maximum absorption around $75 \mathrm{GHz}$. The tolerance of the silicon wafer thickness is responsible for the frequency shift, and its real tested value has been updated in the simulations.
Moreover, these measurement results have been compared with the simulated absorption of all LEKID components, in order to obtain the absorption efficiency. Fig. 19(a) and Fig. 19(b) show a comparison between test results and simulations of each component, which confirms that incident power is mainly absorbed in the inductor, as expected. The inductor absorption efficiency is reduced due to the dielectric loss, which has a negligible effect at cryogenic temperature.

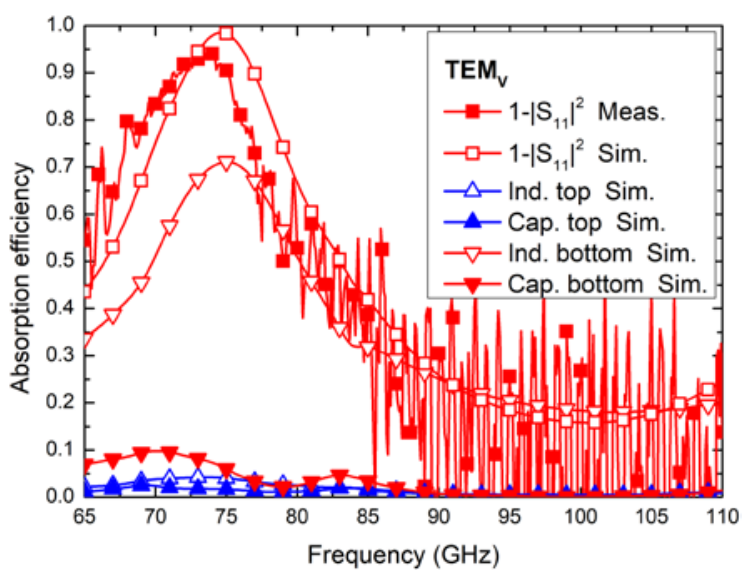

(a)

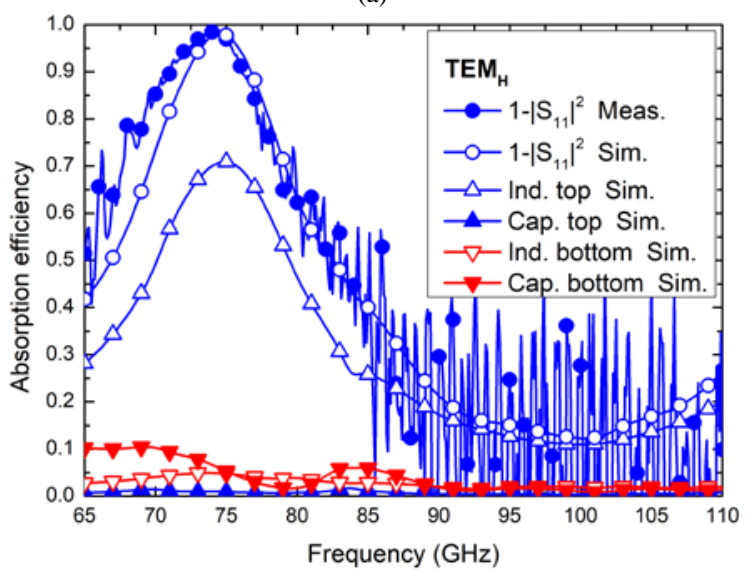

(b)

Fig. 19. Absorption measurements and simulations of $11 \times 11$ LEKIDs at Wband for two orthogonal polarizations at ambient temperature; (a) TEM $\mathrm{V}$ wave, with maximum absorption in the LEKID at the bottom. (b) $\mathrm{TEM}_{\mathrm{H}}$ wave with maximum absorption in the LEKID at the top.

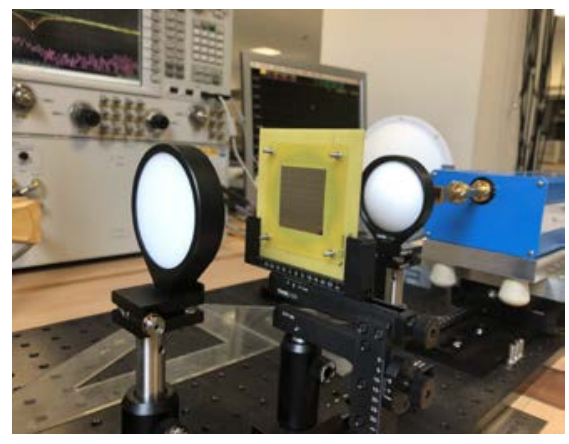

Fig. 20. Dual-polarization LEKID array under absorption test at W-band. 


\section{B. Cryogenic Measurements.}

Cryogenic characterization of the 7 pixels array was performed using the experimental set-up explained in Section IV.B. A Vector Network Analyzer is employed for measuring the $S_{21}$ parameter across the array. Fig. 21 shows the $\mathrm{S}_{21}$ transmission amplitude through the array, where each minimum corresponds to a pixel. Due to the low pixel packing density in this prototype, negligible crosstalk between resonators is expected [31]. The resonant frequency, loaded quality factor $(Q)$, external quality factor $\left(Q_{c}\right)$ and internal quality factor $\left(Q_{i}\right)$ was obtained following the procedure detailed in [32].

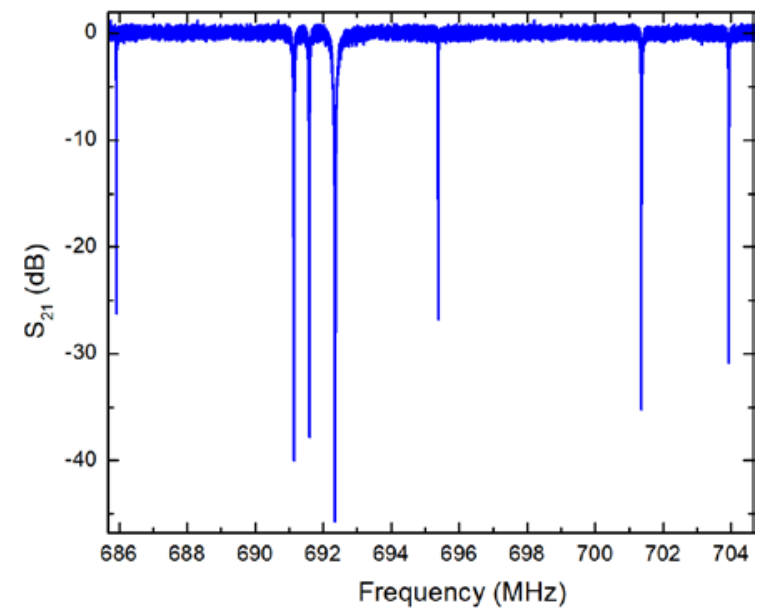

Fig. 21. Transmission sweep $\left(\mathrm{S}_{21}\right)$ measured for the fabricated Ti/Al LEKID array at $\mathrm{T}=12 \mathrm{mK}$ and dark environment.

The EM simulation software Sonnet was used to confirm the estimated kinetic inductance $\left(\mathrm{L}_{\mathrm{k}}\right)$ from electrical characterization [17]. Fig. 22 shows the simulated resonant frequency of the lowest resonance as a function of the kinetic inductance. As can be seen, the experimental resonant frequency corresponds to $\mathrm{L}_{\mathrm{k}}=2.2 \mathrm{pH} / \mathrm{sq}$, very close to the estimated value. These simulations estimate the kinetic fraction by comparing the experimental resonance and the simulated one, obtaining $\alpha=0.38$.

The quality factors obtained for all of the pixels are shown in Fig. 23. The internal quality factor, around $10^{6}$ for all the pixels, indicates the excellent quality of the deposited film [33]. The external quality factor, as can be seen, has been tuned within an order of magnitude, following the procedure explained in Section II.B. The lowest value, $3.5 \cdot 10^{3}$ corresponds to the pixel shown in Fig. 15(a), whereas the highest value, $5 \cdot 10^{4}$, corresponds to the pixel shown in Fig. 15(b). The obtained external quality factors agree reasonably well with the values from the design methodology. Differences between measurement and simulation are related to small tolerances in the nanofabrication process.

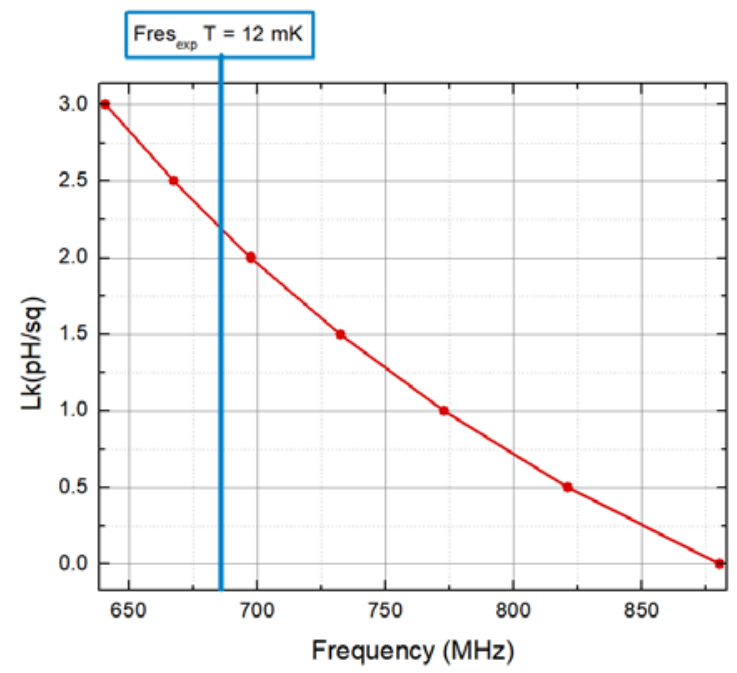

Fig. 22. Simulated resonant frequency as a function of the kinetic inductance. Vertical blue line indicates the measured experimental resonant frequency at $12 \mathrm{mK}$. Crossover between this line and the simulated values (red) shows the obtained kinetic inductance $2.2 \mathrm{pH} / \mathrm{sq}$.

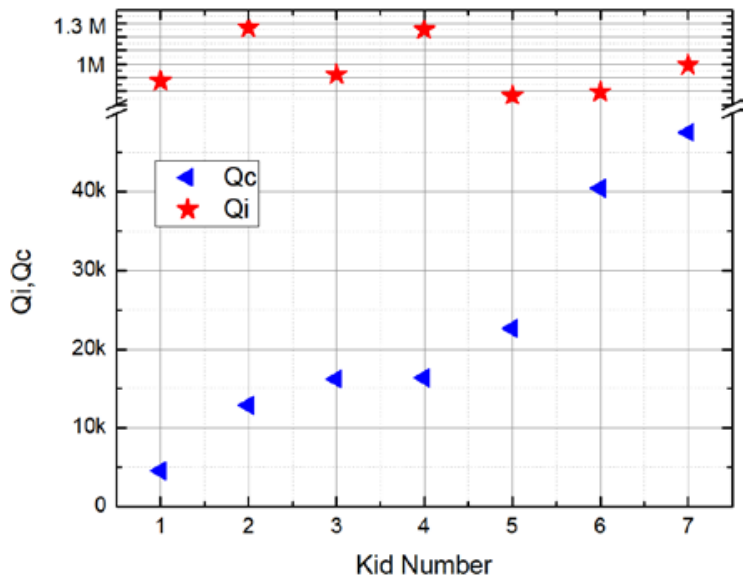

Fig. 23. Quality factor distribution for the 7 pixels fabricated, obtained at $\mathrm{T}=12 \mathrm{mK}$ and dark environment. The obtained $Q_{c}$ values have been ordered from lowest to highest for clarity.

Under these dark conditions, the external quality factor limits the loaded quality factor (overcoupling, $Q_{i}>Q_{c}$ ). However, under the optical load, the internal quality factor is expected to diminish, leading the LEKIDs to a critical optical coupling $\left(Q_{c}\right.$ $=Q_{i}$ ) which is desirable for maximizing the response [16]. For simulating this effect, a temperature sweep has been performed as shown in Fig. 24(a). Fig. 24(b) shows the loaded, internal and external quality factors as a function of bath temperature. As can be seen, $Q_{i}$ is reduced as temperature is increased due to thermally excited quasiparticles. This effect can be compared with the response of the LEKIDs upon optical illumination [34]. As can be seen, the LEKID goes from an overcoupled to undercoupled regime, showing the importance of a proper microwave design for reaching the critical coupling $\left(Q_{i}=Q_{c}\right)$. Future experiments will be performed under optical illumination in order to choose the optimum external coupling. 


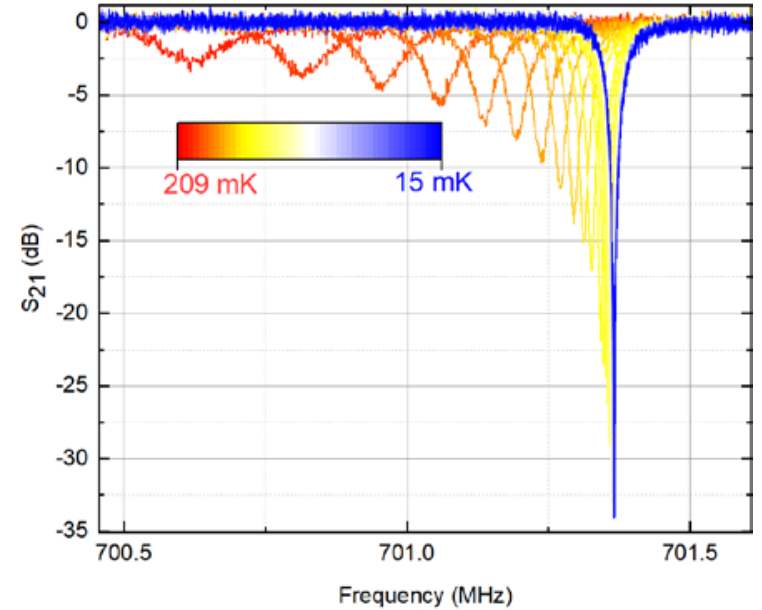

(a)

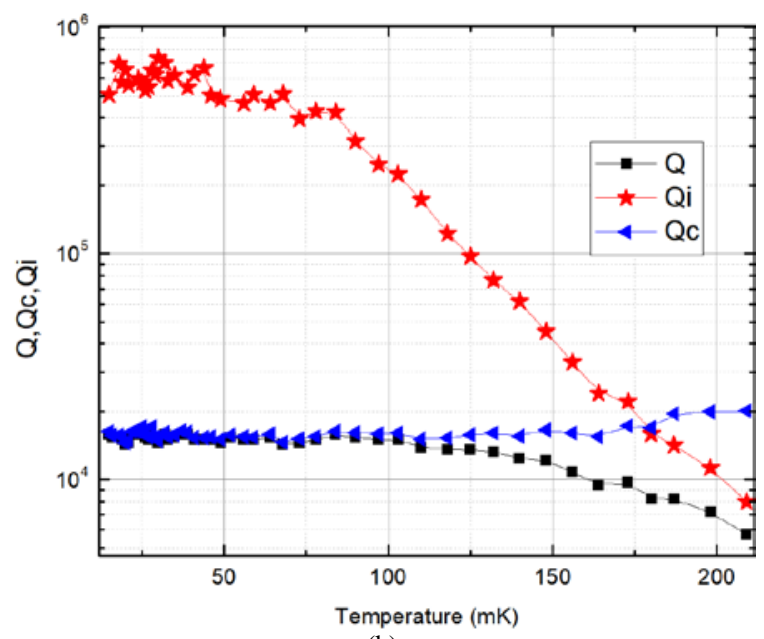

(b)

Fig. 24. (a) Transmission $\left(\mathrm{S}_{21}\right)$ measured for a fabricated Ti/Al LEKID versus temperature. (b) Loaded, internal and external quality factors as a function of bath temperature. A crossover from overcoupling to undercoupling can be observed.

\section{CONCLUSION}

This work has demonstrated dual-polarization LEKID array absorption at W-band at ambient temperature. A superconducting $\mathrm{Ti} / \mathrm{Al}$ bilayer is used to push down the critical temperature (from $1.2 \mathrm{~K}$ of pure $\mathrm{Al}$ to $782 \mathrm{mK}$ ), and therefore push down the low frequency limit imposed by the superconducting gap to this frequency band. The used topology allows us to detect simultaneously two orthogonal linearly polarized waves in one single pixel. The described design methodology is applicable to other millimeter and submillimeter wave bands.

On the other hand, a prototype with seven pixels was fabricated adjusting the external coupling factor, following a proposed method, in order to achieve critical coupling under the desired optical load. The results obtained exhibit a high internal quality factor. The range of the external quality factors applied has experimentally demonstrated a critical coupling when the bath temperature is increased to emulate an optical load. The dual-polarization LEKID design presented and its initial test results show a very promising technology for future polarimetry experiments.

\section{ACKNOWLEDGMENT}

We acknowledge M. Calvo and A. Monfardini, both from Institut Néel and Université Grenoble Alpes CNRS, Grenoble (France), for useful discussions.

\section{REFERENCES}

[1] Monfardini, L.J. Swenson, A. Bideaud, et al., "NIKA: A millimeter-wave kinetic inductance camera," A\&A, vol. 521, A29, June 2010, doi: 10.1051/0004-6361/201014727.

[2] P. K. Day, H.G. LeDuc, B.A. Mazin, A. Vayonakis, J. Zmuidzinas, "A broadband superconducting detector suitable for use in large arrays," Nature, vol. 425, pp. 817-821, Oct. 2003, doi:10.1038/nature02037.

[3] P. Szypryt, "Development of Microwave Kinetic Inductance Detectors for Applications in Optical to Near-IR Astronomy,” Ph.D. dissertation, Univ. California Santa Barbara, 2017.

[4] N. Boudou et al., "Kinetic inductance detectors for millimeter and submillimeter astronomy," Comptes Rendus Physique, vol. 13, no. 1, pp. 62-70, Jan-Feb. 2012, doi: 10.1016/j.crhy.2011.10.008.

[5] A. Catalano, J. Goupy, H. le Sueur, A. Benoit, O. Bourrion, M. Calvo, L. Dumoulin, F. Levy-Bertrand, J. Macìas-Pérez, S. Marnieros, N. Ponthieu, A. Monfardini, "Bi-layer kinetic inductance detectors for space observations between 80-120 GHz," A\&A, vol. 580, no. A15, Aug. 2015, doi: 10.1051/0004-6361/201526206.

[6] M. Roesch, et al., "Development of Lumped Element Kinetic Inductance Detectors for NIKA," in Proc. Int. Symp. on Space THz Techol., Tucson, Arizona, USA, Apr. 2011.

[7] J. van Rantwijk, M. Grim, D. van Loon, S. Yates, A. Baryshev, J. Baselmans, "Multiplexed Readout for 1000-Pixel Arrays of Microwave Kinetic Inductance Detectors," IEEE Trans. Microw. Theory Techn., vol. 64, no. 6, pp. 1876-1883, June 2016, doi: 10.1109/TMTT.2016.2544303.

[8] H. McCarrick, et al., "Design and performance of dual-polarization lumped-element kinetic inductance detectors for millimeter-wave polarimetry," A\&A, vol. 610, no. A45, Feb. 2018, doi: 10.1051/00046361/201732044.

[9] L. Perotto, et al., "Calibration and performance of the NIKA2 camera at the IRAM 30-m Telescope,” A\&A, vol. 637, no. A71, May 2020, doi: 10.1051/0004-6361/201936220.

[10] A. Paiella, et al., "Kinetic inductance detectors for the OLIMPO experiment: design and pre-flight characterization," J. Cosmology Astroparticle Phys., vol. 2019, Jan. 2019, doi: 10.1088/14757516/2019/01/039.

[11] S. Doyle, "Lumped element Kinetic Inductance Detectors," PhD Thesis, Cardiff University, U.K., 2008.

[12] A. Catalano, et al., "Maturity of lumped element kinetic inductance detectors for space-borne instruments in the range between 80 and 180 GHz," A\&A, vol. 592, no. A26, Aug. 2016, doi: 10.1051/00046361/201527715.

[13] A. Catalano, et al., "Sensitivity of LEKID for space applications between $80 \mathrm{GHz}$ and $600 \mathrm{GHz}$,” A\&A, vol. 641, no. A179, Sep. 2020, doi: 10.1051/0004-6361/202038199.

[14] J. Perido, J. Glenn, P. Day, et al., "Extending KIDs to the Mid-IR for Future Space and Suborbital Observatories,” J. Low Temp. Phys., vol. 199, pp. 696-703, Feb. 2020, doi; 10.1007/s10909-020-02364-y.

[15] A. Gomez, A., M. Calvo, J. Goupy, et al., "Polarization Filter for Microstrip Lumped-Element Kinetic Inductance Detectors,” J. Low Temp. Phys., vol. 193, pp. 157-162, Jul. 2018, doi: 10.1007/s10909-0182021-1.

[16] J. Zmuidzinas, "Superconducting Microresonators: Physics and Applications," Annual Rev. Condensed Matter Phys., vol. 3, pp. 169-214, Mar. 2012, doi 10.1146/annurev-conmatphys-020911-125022.

[17] B. Aja, L. de la Fuente, A. Fernandez, Juan P. Pascual, E. Artal, M. C. de Ory, M. T. Magaz, D. Granados, J. Martin-Pintado, A. Gomez, "Bi-Layer Kinetic Inductance Detectors for W-Band," in IEEE Int. Microw. Symp., Virtual Conf., Aug. 4-6, 2020.

[18] M. Roesch, "Development of Lumped Element Kinetic Inductance Detectors for Mm-Wave Astronomy at the IRAM 30 m Telescope," Ph.D. dissertation, KIT, Germany, doi: 10.5445/KSP/1000036607.

[19] R. Ulrich, "Far-Infrared Properties of Metallic Mesh and its Complementary Structure," Infrared Phys., vol. 7, pp. 37-55, Mar. 1967, doi: 10.1016/0020-0891(67)90028-0. 
[20] R. Ulrich, T.J. Bridges, M.A. Pollack, "Variable Metal Mesh Coupler for Far Infrared Lasers,” Appl. Opts., vol. 9, no. 11, pp. 2511-2516, Nov. 1970, doi: 10.1364/AO.9.002511.

[21] N. Marcuvitz, "Gratings and Arrays in Free Space," in Waveguide Handbook. New York, NY, USA: McGraw-Hill, 1951, pp.280-285.

[22] Zhang, Y., Wang, Z., "Measurement of dielectric loss tangent at cryogenic temperature using superconducting film resonator," J. Theor. Appl. Phys., vol. 10, pp.27-32, 2016, doi: 10.1007/s40094-015-0197-1.

[23] S. Shu et al., "Optical Response of Lumped-Element Kinetic-Inductance Detector Arrays," IEEE Tran. THz Sci. Technol., vol. 8, no. 6, pp. 605612, Nov. 2018, doi: 10.1109/TTHZ.2018.2873127.

[24] S. Wuensch, G. Hammer, T. Kappler, F. Geuppert, M. Siegel, "Investigation and Optimization of LEKID Coupling Structures and Multi-Pixel Arrays at 4.2 K,” IEEE Trans. Appl. Supercond., vol. 21, no. 3, pp. 752-755, June 2011, doi: 10.1109/TASC.2010.2090445.

[25] R. Bojanic, V. Milosevic, B. Jokanovic, F. Medina-Mena, F. Mesa, "Enhanced Modelling of Split-Ring Resonators Couplings in Printed Circuits," IEEE Trans. Microw. Theory Techn., vol. 62, no. 8, pp.16051615, Aug. 2014, doi: 10.1109/TMTT.2014.2332302.

[26] A. Khanna and Y. Garault, "Determination of Loaded, Unloaded, and External Quality Factors of a Dielectric Resonator Coupled to a Microstrip Line," IEEE Trans. Microw. Theory Techn., vol. 31, no. 3, pp. 261-264, Mar. 1983, doi: 10.1109/TMTT.1983.1131473.

[27] J. Reed and G. J. Wheeler, "A Method of Analysis of Symmetrical FourPort Networks," IRE Trans. Microw. Theory Techn., vol. 4, no. 4, pp. 246252, Oct. 1956, doi: 10.1109/TMTT.1956.1125071.

[28] V. K. Tripathi and Y. K. Chin, "Analysis of the general nonsymmetrical directional coupler with arbitrary terminations," IEE Proc. $H$ Microwaves, Optics and Antennas, vol. 129, no. 6, pp. 360-362, Dec. 1982, doi: 10.1049/ip-h-1.1982.0072.

[29] O. Garcia, F. Tercero and S. Lopez, "Free-space W-band Setup for the Electrical Characterization of Materials and Mm-Wave Components," CDT Technical Report 2017-9, Sep 2017. [Online]. Available: http://www1.oan.es/reports/doc/IT-CDT-2017-9.pdf.

[30] P.F. Goldsmith, Quasioptical Systems: Gasussian Beam Quasioptical Propagation and Applications. Piscataway, NJ : IEEE Press, 1998.

[31] O. Noroozian, P. K. Day, B. H. Eom, H. G. Leduc and J. Zmuidzinas, "Crosstalk Reduction for Superconducting Microwave Resonator Arrays," IEEE Trans. Microw. Theory Techn., vol. 60, no. 5, pp. 12351243, May 2012, doi: 10.1109/TMTT.2012.2187538.

[32] S. Probst, F. B. Song, P. A. Bushev, A. V. Ustinov, and M. Weides, "Efficient and robust analysis of complex scattering sata under noise in microwave resonators,” Rev. Sci. Instrum., vol. 86, Feb. 2015, doi: 10.1063/1.4907935.

[33] B. A. Mazin, ”Superconducting Materials for Microwave Kinetic Inductance Detectors," 2020, arXiv:2004.14576.

[34] R. M. J. Janssen, et al., "Equivalence of optical and electrical noise equivalent power of hybrid NbTiN-Al microwave kinetic inductance detectors," Appl. Phys. Lett., vol. 105, no. 19, Nov. 2014, doi: $\underline{10.1063 / 1.4901733 .}$.

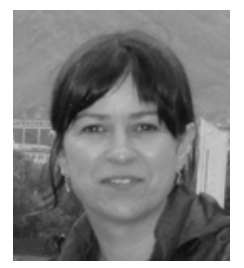

Beatriz Aja received the Telecommunications Engineering degree and the Ph.D. in 1999 and 2007 respectively from the University of Cantabria, Spain.

She is currently an Associate Professor with the Department of Communications Engineering at the University of Cantabria, Spain.

From 2008 to 2012 and from 2013 to 2015 she was invited scientist at the Fraunhofer Institute for Applied Solid State Physics (Germany), in a joint collaboration project with Centro Astronómico de Yebes (Spain), where her main activity was the design of Monolithic Microwave Integrated Circuits (MMIC) for cryogenic applications. She has been involved in development of the receivers for the Planck Mission and QUIJOTE (Q-U-I JOint Tenerife) CMB Experiment. Her research interests include design and characterization of LNAs for cryogenic applications, microwave and millimeter-wave passive components and radio astronomy receivers.

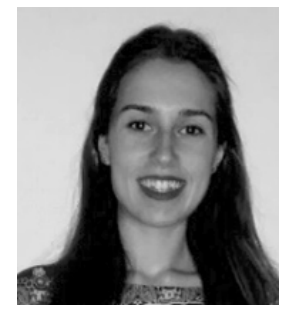

Marina C. de Ory was born in Seville, Spain, in 1994. She obtained her Bachelor of Science and Material Engineering from Seville University in 2017. She completed her Master of NanoScience and Advanced Materials at Universidad Complutense de Madrid, Spain, in 2018.

She joined the Department of Transport and Quantum Phenomena at Complutense University of Madrid as a research assistant in 2018, where she was involved in superconductor transport measurements. Then, she joined Centro de Astrobiología (CSIC-INTA) in collaboration with IMDEA-Nanoscience, Madrid, where she is currently pursuing the Ph.D degree in the field of low temperature physics, ultrasensitive detectors for astronomy and quantum technologies. Her research interests lie in the area of superconducting resonators, including modeling, nanofabrication and low temperature microwave characterization of microwave devices with applications in Astrophysics and Quantum Computing. She is also involved in the study of the interaction of superconductors with 2D and magnetic materials. She collaborates actively with other researchers in the same field and contributes to several scientific conferences.

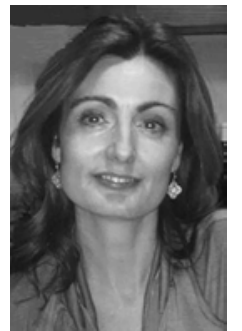

Luisa de la Fuente (Member, IEEE) received the M.Sc. degree in physics and the Ph.D. degree in Electronic Engineering from the University of Cantabria, Santander, Spain in 1991 and 1997, respectively.

In 1992 she joined the Department of Communications Engineering at the University of Cantabria, where she is currently an Associate Professor. Her main research interests include design and testing of microwave circuits in both hybrid and monolithic technologies. In the last years she has worked in projects focused in the development of radiometers for space applications, like the Planck Mission, in particular in low noise amplifiers at room and cryogenic temperatures. Currently she is involved in several projects focused on the development of very low noise receivers in the 30 and $40 \mathrm{GHz}$ frequency bands for the QUIJOTE experiment, and on the development of polarimetry receivers at $\mathrm{W}$ band.

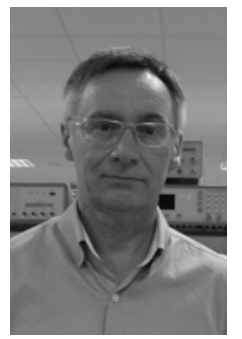

Eduardo Artal (M’81) (LM'19) received the Engineer and Dr. Engineer in Telecommunication degrees from the Technical University of Catalonia, Barcelona, Spain, in 1976 and 1982, respectively. From 1976 to 1990 he was an Assistant Professor with the Technical University of Catalonia. From 1979 to 1981, in a partial leave from the university, he joined Mier Allende S.A., Barcelona, Spain, where he was involved with TV and FM radio reemitters development. Since 1990 he has been a Professor at the University of Cantabria, Santander, Spain, where he was manager of the Telecommunication Engineering course from 1990 to 1994. From 1994 to 1998 he was manager of the National Program for Information and Communications Technologies at the "Plan Nacional de I+D”, National R\&D Plan in the Spanish Ministry of Education and Science, Madrid. His main areas of activities and contributions have been microwave circuits and systems, including monolithic microwave integrated circuits up to $50 \mathrm{GHz}$. His current research interests are low noise millimeter-wave amplifiers and receivers for radio-astronomy applications.

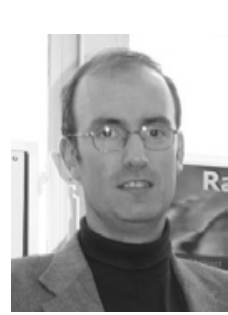

Juan Pablo Pascual (Senior Member, IEEE) was born in Santander (Spain) in 1968. M. Degree in Electronics with honors (1990) and Ph.D. degree in Electronic Engineering (1996), from the University of Cantabria, where he works currently as associated professor at the Communications Engineering Department. He is senior member of the IEEE. His research interests are active device modelling, MMIC design methodology of nonlinear functions and subsystems, from microwaves to millimeter waves and Terahertz, and system simulation. He has co-authored over than 60 contributions in international journals and congress. He has been involved in modelling and design projects with institutions like Alcatel Espacio, OMMIC, ESA, Technical University of Darmstadt (where he stayed during 1999), the PLANCK, the QUIJOTE mission, and the "Terahertz Technology for Electromagnetic Sensing Applications" consortiums. He has participated in the Spanish Network of 
Excellence in Terahertz and has been responsible for a training program of Doctors for the industry (University of Cantabria-Erzia Tech).

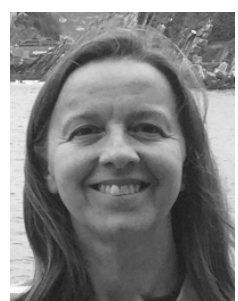

María Teresa Magaz was born in Madrid, Spain in 1965. She obtained her BSc (1986) and MSc (1988) degrees in Organic Chemistry from Universidad Autónoma de Madrid and the MSc degree in Occupational Health and Safety in 2010.

She was a Research Assistant Technician in La Marañosa, Institute of Technology (INTA) from 2010 to 2015 in Madrid, working on optical photolithography for the development of PbSe IR detectors. Since 2017 she has been working as a Research Assistant Technician in the Centro de Astrobiología (CSIC-INTA) in collaboration with IMDEA-Nanociencia in Madrid. Her research focuses on processing and optimization of nano and microfabrication of superconductor Lumped Element Kinetic Inductance Detectors for future space missions. Her expertise includes sputtering deposition in high vacuum conditions, optical and electrical lithography and reactive ion etching techniques.

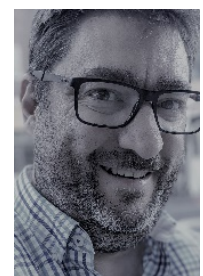

Daniel Granados was born in Madrid, Spain in 1977. He obtained his BSc (2001) and MSc (2002) in Physics from the Universidad Autónoma de Madrid (Spain). He obtained his PhD in 2006 working at the Spanish Research Council (IMM-CNM-CSIC). In 2005 he was visiting scientist at the Nano-Optics group at Heriot-Watt University, Edinburgh (UK). He joined the Quantum Information Group at Toshiba Research Europe Ltd, Cambridge (UK) in 2006. During this time, he was also visiting scientist and collaborator of the Semiconductor Physics Group at the Cavendish Laboratory, Cambridge (UK).

He joined IMDEA-Nanoscience in Madrid in September 2009. He is Executive Director of Scientific Infrastructures and group leader of the Quantum nanoDevices Group. The group has varied interests in nanofabrication, single-photon emitters, superconductor detectors, devices based on 2D materials, photonics, nano-optics, quantum information technologies and cavity quantum electrodynamics.

Dr. Granados has published more than 80 peer-reviewed works, has attended $>30$ international conferences and contributed to $>75$. In the last 5 years, he has imparted $>30$ invited seminars at universities and research centres on Europe and the US. He has $>2000$ citations, an h factor of 20 and i10 factor of 37 . He has been PI in 17 research projects and contributed to more than 30 .

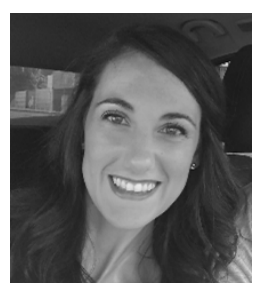

Alicia Gomez was born in Madrid, Spain in 1986. She obtained her BSc (2009) and MSc (2010) in Physics from the Universidad Complutense de Madrid (Spain). She obtained her PhD from Universidad Complutense of Madrid in 2013, focused on superconducting vortex dynamics.

She was a visiting researcher in University California-Davis (US) in 2010 and in University of Antwerpen (Belgium) in 2012. Since 2013, she is a postdoctoral researcher in Centro de Astrobiología (CSIC-INTA) where she is in charge of the development of superconducting kinetic inductance detectors for space and ground based instrumentation. She is member of international collaborations such as the NIKA2 Camera installed at the IRAM $30 \mathrm{~m}$ telescope in Granada and the KISS Camera for the Canary Island telescope. She was also part of the CORE proposal submitted to the call for the M5 mission of the ESA Cosmic Vision. She has authored or coauthored more than 40 peer-reviewed papers. Her experience spans from the design and fabrication of superconducting electronics in cleanroom to the electronic characterization of superconducting devices at cryogenic temperatures with and without magnetic fields.

Dr. Gomez was awarded with a Spanish MICINN Juan de la CiervaIncorporación postdoctoral fellowship in 2018 to lead the development of Kinetic Inductance Detectors at Centro de Astrobiología (CSIC-INTA), Spain for two years. 